\title{
Burden of diarrhea in the Eastern Mediterranean Region, 1990-2015: Findings from the Global Burden of Disease 2015 study
}

\author{
GBD 2015 Eastern Mediterranean Region Diarrhea Collaborators ${ }^{1}$
}

Received: 30 April 2017/Revised: 20 June 2017 / Accepted: 28 June 2017/Published online: 3 August 2017

(C) The Author(s) 2017. This article is an open access publication

\begin{abstract}
Objectives Diarrheal diseases (DD) are an important cause of disease burden, especially in children in low-income settings. DD can also impact children's potential livelihood through growth faltering, cognitive impairment, and other sequelae.

Methods As part of the Global Burden of Disease study, we estimated DD burden, and the burden attributable to specific risk factors and etiologies, in the Eastern Mediterranean Region (EMR) between 1990 and 2015. We calculated disability-adjusted life-years (DALYs) - the sum of years of life lost and years lived with disability-for both sexes and all ages.

Results We estimate that over 103,692 diarrhea deaths occurred in the EMR in 2015 (95\% uncertainty interval: $87,018-124,692)$, and the mortality rate was 16.0 deaths per 100,000 persons (95\% UI: 13.4-19.2). The majority of these deaths occurred in children under 5 (63.3\%) $(65,670$
\end{abstract}

This article is part of the supplement "The state of health in the Eastern Mediterranean Region, 1990-2015".

The members of GBD (Global Burden of Disease) 2015 Eastern Mediterranean Region Diarrhea Collaborators are listed at the end of the article. Ali H. Mokdad, on behalf of GBD 2015 Eastern Mediterranean Region Diarrhea Collaborators, is the corresponding author.

Electronic supplementary material The online version of this article (doi:10.1007/s00038-017-1008-z) contains supplementary material, which is available to authorized users.

GBD 2015 Eastern Mediterranean Region Diarrhea

Collaborators

mokdaa@uw.edu

1 Institute for Health Metrics and Evaluation, 2301 5th Ave, Suite 600, Seattle, WA 98121, USA deaths, 95\% UI: 53,640-79,486). DALYs per 100,000 ranged from 304 (95\% UI 228-400) in Kuwait to 38,900 (95\% UI 25,900-54,300) in Somalia.

Conclusions Our findings will guide evidence-based health policy decisions for interventions to achieve the ultimate goal of reducing the DD burden.

Keywords Eastern Mediterranean Region - Burden of disease $\cdot$ Diarrheal diseases

\section{Introduction}

Rigorous public health efforts resulted in a significant decline in mortality due to diarrheal diseases (DD) over the past 20 years. However, these diseases continue to cause a major global disease burden, especially in children under 5 years of age. In addition, the incidence of childhood diarrhea in low-income countries has not declined as rapidly as mortality (GBD 2015 Risk Factors Collaborators 2016). In the most recent Global Burden of Disease (GBD) study, DD was the fourth-leading cause of death among children under 5, responsible for 499,000 deaths (95\% UI: $447,000-558,000$ ), representing $8.6 \%$ of all deaths in this age group (GBD 2015 Risk Factors Collaborators 2016). For those who survive these illnesses and suffer from repeated infections by enteric pathogens during the critical early years of life; DD can lead to serious, lifelong health consequences such as environmental enteric dysfunction (EED), growth faltering, impaired cognitive development, and reduced immune response to infection and vaccinations (Guerrant et al. 2013).

DD pathogen etiologic contribution may vary depending on the study's geographic location, duration, or the population sampled (Lindsay et al. 2015). These infections are 
believed to be different in the developing world compared to the developed world with regard to a number of features, including earlier age of onset, multiple repeated exposures, greater diversity of pathogens, nutritional status of the host, and a number of others, such as co-infection, diet, and genetics (Heidt et al. 2014).

The Eastern Mediterranean Region (EMR) is home to more than 500 million people, representing a diverse group of 22 countries. EMR countries have diverse historical backgrounds, political and social contexts, and fiscal and cultural influences on their health care systems. The region has wide variation in per capita gross national product (GNP) (The World Bank 2016), which has a major influence on overall health spending and results in substantial health inequities both within and across countries. During recent years DD prevention efforts that focus on vaccines in the short term and improvements in water, sanitation, and hygiene in the long term have been impeded by warfare and political unrest in the region. These conflicts and wars also resulted in a huge problem of internal displacement and refugees (Mokdad et al. 2016).

Many countries in the EMR achieved important successes in the fight against DD in the 1970s and 1980s with the support of United Nations International Children's Emergency Fund (UNICEF) and the World Health Organization (WHO) through the National Control of Diarrheal Diseases Project (NCDDP) (WHO 1992; Enzley et al. 1997). For example, Egypt's program, which spanned from 1981 to 1991 , was credited with significantly improving diarrheal case management (National Control Of Diarrheal Diseases Project 1988; El-Rafie et al. 1990; Cobb et al. 1996). However, over the last two decades, momentum has slowed (Forsberg et al. 2007). As of the date of this report, rotavirus vaccines have been introduced through National Immunization Programs in only nine countries in the region: Djibouti, Jordan, Libya, Morocco, Qatar, Saudi Arabia, Sudan, United Arab Emirates, and Yemen (PATH 2017).

In this report, we are updating our previous burden estimates (Khalil et al. 2016), pathogen distribution, and risk factors for diarrhea in children and adults in the EMR for 1990-2015.

\section{Methods}

The Global Burden of Disease Study (GBD 2015) is a systematic, comprehensive effort to quantify health loss from more than 300 diseases and injuries, including diarrheal diseases and associated risk factors. The GBD estimation strategy, including for diarrheal diseases, has been described in detail elsewhere (Foreman et al. 2012; Flaxman et al. 2015). The burden of diarrheal diseases is measured in deaths, incidence, and disability-adjusted lifeyears (DALYs), which are the sum of years of life lost (YLLs) and years lived with disability (YLDs). The etiological burden was also estimated for 13 pathogens associated with diarrhea.

All estimates are produced by year and by age, for both sexes, and for all countries. In accordance with the guidelines for accurate and transparent health estimates reporting (GATHER), code for each step of the estimation process is available online on GitHub (http://www.ghdx. healthdata.org/gbd-2015-code) (Institute for Health Metrics and Evaluation). The methods of each of these steps are summarized below.

\section{Study region}

The EMR countries were grouped according to per capita gross national income (GNI) into low-income countries (LICs) [Islamic Republic of Afghanistan (Afghanistan), Djibouti, Somalia, Republic of Yemen (Yemen)]; middleincome countries (MICs) [Arab Republic of Egypt (Egypt), Islamic republic of Iran (Iran), Iraq, Jordan, Lebanon, Libya, Morocco, Pakistan, Palestine, Sudan, Syrian Arab Republic (Syria), Tunisia]; and high-income countries (HICs) [Bahrain, Kuwait, Oman, Qatar, Saudi Arabia, and the United Arab Emirates (UAE)]. We defined LICs as those having a per capita GNI of $\$ 1045$ or less, MICs as those with a per capita GNI between $\$ 1046$ and $\$ 12,735$, and HICs as countries with per capita GNI of $\$ 12,736$ or greater (The World Bank 2016).

\section{Mortality}

Cause-specific mortality estimates for diarrheal diseases were modeled using a Bayesian ensemble modeling process (GBD 2015 Mortality and Causes of Death Collaborators 2016). Diarrhea mortality data included vital registration and verbal autopsy sources. The modeling process estimated the mortality rate due to diarrhea for both sexes from 1990 to 2015 for all age groups in every country and subnational regions in select countries. We considered the following covariates: education, lag-dependent income, underweight, latitude, population density, improved water and sanitation sources, diarrhea risk factors summary, Socio-demographic Index (SDI), and rotavirus vaccine coverage. The ensemble model approach allows for a suite of models, weighted by out-of-sample predictive validity, to inform the final estimates.

\section{Morbidity}

Diarrheal cases were defined as three or more loose stools in a 24-h period. As with mortality, morbidity was modeled 
at every year, sex, age, and geographic location in GBD 2015. The morbidity model used DisMod-MR 2.1, a Bayesian meta-analytic, age-integrating, mixed-effects model which is available online on EpiViz (http://www. vizhub/healthdata.org/epi) (Kassebaum et al. 2016). Diarrhea prevalence and incidence data from a systematic literature review, population-representative surveys, and hospital and health care utilization data informed the nonfatal model.

\section{Etiologies}

Diarrhea cases and deaths were attributed to pathogens using a counterfactual approach that accounts for exposure to pathogens and for the association between each pathogen and diarrhea. A systematic literature review on the proportion of diarrhea cases that test positive for a set of pathogens was updated for GBD 2015. These data were used in the DisMod-MR framework to estimate the age, sex, year, and geographic distribution of pathogens in diarrheal episodes. The population attributable fraction (PAF) was used to identify the fraction of diarrhea cases and deaths due to each pathogen. The PAF was calculated as: (GBD 2015 Mortality and Causes of Death Collaborators 2016).

$\mathrm{PAF}=$ Proportion $\times\left(1-\frac{1}{\mathrm{OR}}\right)$,

where Proportion is the proportion of cases positive for a pathogen and the odds ratio (OR) is the odds of diarrhea given pathogen detection. The odds ratios were from a systematic reanalysis of the Global Enteric Multicenter Study (GEMS) (Kotloff et al. 2013), a multi-site casecontrol study of moderate-to-severe diarrhea in children under 5 that systematically tested nearly half of the original GEMS samples using a molecular quantitative polymerase chain reaction (qPCR) diagnostic (Liu et al. 2016). A mixed-effects conditional logistic regression model estimated the odds ratios for diarrhea including random effects on site to account for geographic variation.

Since the odds of diarrhea given pathogen presence were calculated using the qPCR diagnostic, we adjusted our proportion estimates to be comparable to qPCR-based estimates. The sensitivity and specificity of the nonmolecular diagnostic techniques from GEMS were evaluated compared to the qPCR diagnostic, and these values were used to make this adjustment with this formula (Wickham 2009):

Proportion $_{\text {True }}=\frac{\left(\text { Proportion }_{\text {Observed }} \times \text { Specificity }-1\right)}{(\text { Sensitivity }+ \text { Specificity }-1)}$.

Vibrio cholerae and Clostridium difficile were estimated separately from the other pathogens in GBD. Cholera cases were estimated using proportion data from published studies to calculate an expected number of annual cases for each country and year, and those estimates were compared to the World Health Organization case notification data (WHO 2016) to estimate underreporting of cholera. Cholera deaths were estimated using case fatality data in DisMod-MR. Since $C$. difficile is frequently associated with hospital and health care utilization, hospital incidence data were modeled in DisMod-MR using hospital and health care utilization data with ICD codes for $C$. difficile.

All etiologies were estimated independently and for each year, geography, age, and sex.

\section{Risk factors}

We also assessed diarrheal DALYs, YLLs, and YLDs attributable to childhood stunting, suboptimal breastfeeding, vitamin A deficiency, zinc deficiency, and water, sanitation, and hygiene (WASH). Risk factor attribution follows a general counterfactual approach where the exposure and relative risk of diarrhea were used to estimate the burden of the aforementioned risk factors (GBD 2015 Risk Factors Collaborators 2016).

\section{Socio-demographic Index}

We evaluate associations between diarrhea and socio-demographic status using the Socio-demographic Index (SDI). The SDI is a composite measure developed for GBD 2015 that accounts for fertility rate, lag-dependent income per capita, and education (GBD 2015 Mortality and Causes of Death Collaborators 2016). To capture the average relationships for each age-sex group, we applied a simple least squares spline regression of the diarrhea mortality rate on SDI. The predicted diarrhea mortality rates from this regression were used as expected mortality rates based on SDI. The SDI is scaled from 0 to 1 where 0 represents the lowest possible observed SDI and 1 is the highest. SDI in 2015 in the EMR ranged from 0.27 in Somalia to 0.83 in Kuwait.

\section{Results}

There were 103,692 diarrhea deaths in the EMR in 2015 (95\% uncertainty interval (UI): 87,018-124,692), and the mortality rate was 16.0 deaths per 100,000 persons $(95 \%$ UI: 13.4-19.2). The majority of these deaths occurred in children under 5 years old (63.3\%) (65,670 deaths, 95\% UI: $53,640-79,486$ ). Although the greatest number of diarrhea deaths occurred in children under 5, diarrhea mortality was also high in the $70+$ year age group. In fact, of the 22 countries in the EMR, the mortality rate was 
Table 1 The number of diarrhea deaths and mortality rate (per 100,000) in 2015 in the Eastern Mediterranean Region for each age group and sex (Global Burden of Disease 2015 study, Eastern Mediterranean Region, 2015)

\begin{tabular}{llcr}
\hline Age & Sex & Deaths & Rate \\
\hline All ages & Both & $103,691.7(87,018.3-124,692.2)$ & $15.99(13.42-19.23)$ \\
All ages & Female & $50,679.8(40,407.1-63,194.3)$ & $16.14(12.87-20.12)$ \\
All ages & Male & $53,011.9(42,617.5-65,304.5)$ & $15.86(12.75-19.53)$ \\
Under 5 & Both & $65,670.3(53,639.7-79,485.9)$ & $81.82(66.83-99.04)$ \\
Under 5 & Female & $34,729.4(26,435.3-45,011.1)$ & $89.09(67.81-115.46)$ \\
Under 5 & Male & $30,940.9(23,892.9-39,342.5)$ & $74.96(57.89-95.32)$ \\
$5-14$ years & Both & $4986.1(3724.4-6501.8)$ & $3.67(2.74-4.78)$ \\
$5-14$ years & Female & $2540.1(1839.4-3414.2)$ & $3.84(2.78-5.16)$ \\
$5-14$ years & Male & $2446(1623.7-3486.2)$ & $3.5(2.32-4.99)$ \\
$15-49$ years & Both & $11,546.4(7917.3-18,792.8)$ & $3.36(2.3-5.47)$ \\
$15-49$ years & Female & $4827.5(3205.6-8471.4)$ & $2.93(1.94-5.13)$ \\
$15-49$ years & Male & $6718.9(4339.3-10,599.2)$ & $3.76(2.43-5.94)$ \\
$50-69$ years & Both & $9457.6(6095.2-15,147.7)$ & $13.21(8.51-21.16)$ \\
$50-69$ years & Female & $3836.5(2060-6875.3)$ & $10.93(5.87-19.58)$ \\
$50-69$ years & Male & $5621.1(3578.4-8787.7)$ & $15.41(9.81-24.08)$ \\
$70+$ years & Both & $12,031.2(9097.8-15,593.3)$ & $70.83(53.56-91.8)$ \\
$70+$ years & Female & $4746.3(3404.3-6304.8)$ & $53.74(38.55-71.39)$ \\
$70+$ years & Male & $7285(5054.7-10,119.4)$ & $89.34(61.99-124.1)$ \\
\hline
\end{tabular}

higher in the $70+$ age group than the under-5 age group in 17 countries. Diarrhea mortality in the elderly was highest in Somalia (1695 per 100,000, 95\% UI: 709-2896), followed by Djibouti and Pakistan (Table 1). Diarrhea mortality decreased over time in the EMR (Table 2; Fig. 1). Between 1990 and 2015, the number of diarrhea deaths decreased by 54\% among all ages (95\% UI: 43-62\%) and $65 \%$ among children under 5 (95\% UI: 55-73\%). The fastest rate of decrease among children under 5 occurred in Iran (97\%, 95\% UI: 93-99\%) and Syria (97\%, 95\% UI: 94-99\%) and the slowest occurred in Somalia (11\%, 95\% UI: -40 to $45 \%)$ and Qatar $(33 \%, 95 \%$ UI: -65 to $72 \%)$.

Diarrhea mortality among children under 5 varied by time and country. The under-5 diarrhea mortality rate was highest in Somalia, followed by Pakistan, Sudan, and Afghanistan (Figs. 2, 3). Due to its high population, the greatest number of under-5 deaths occurred in Pakistan (55,500 deaths, 95\% UI: 43,258-70,027). Diarrhea mortality was associated with trends in the Socio-demographic Index in most geographies (Figs. 2b, 3), but the mortality rate was much lower in Palestine and Iraq than would be expected based on SDI alone. The ratio of the observed mortality rate to the expected mortality rate based on SDI alone was lowest in Syria and Palestine, where mortality was $3 \%$ of the expected value, and highest in Bahrain, where the mortality was $65 \%$ higher than expected based on SDI alone. Syria and Palestine have moderate SDI and very low diarrhea mortality rates.
Diarrhea was also responsible for a large number of illness episodes. In 2015, diarrhea incidence was 2.1 (95\% UI: 1.9-2.4) per child-year, totaling more than 171 million episodes across the EMR. The incidence was much lower in adults, including those $70+$ years old $(0.7$ per personyear, 95\% UI: 0.69-0.75). The case fatality, expressed as the number of deaths over the number of cases, was $0.1 \%$ in those $70+$ years old, significantly greater than in children under 5 years $(0.038 \%)$. Case fatality increased nonlinearly with incidence (e-Figure 1). Despite the large observed reductions in diarrhea mortality between 1990 and 2015, diarrhea incidence decreased much more marginally (20.7\%).

Among diarrheal etiologies, rotavirus was the leading cause of death in 2015 among children under $5(13,180$ deaths, 95\% UI: 9807-17,738), followed by Shigella (10,964 deaths) and enterotoxigenic E. coli (ETEC) $(6885$ deaths) (Table 3 and e-Figure 1). Among all ages, Shigella was the leading cause of diarrheal deaths $(19,450$ deaths, 95\% UI: 10,026-33,996), followed by Aeromonas and rotavirus. Overall, 99\% of diarrheal deaths in 2015 were attributed to at least one etiology (e-Figure 2).

Unsafe water, sanitation, and hygiene were responsible for over 95\% of diarrhea DALYs in the EMR in 2015 (95.1\%, 95\% UI: 89.2-98.1\%), which is comparable with the global total in 2015. The proportion of diarrhea DALYs due to unsafe WASH was lowest in Jordan (87.0\%) and highest in Sudan $(97.0 \%)$ (e-Figure 3). Childhood 


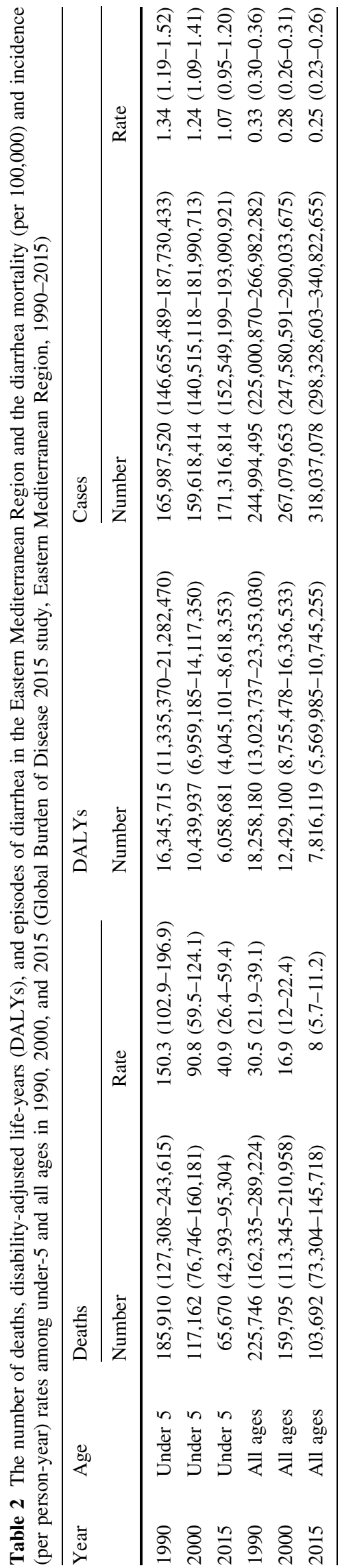

undernutrition was the second-leading risk factor for diarrheal DALYs, responsible for $74.0 \% \quad(95 \%$ UI: $67.4-79.1 \%)$, which is significantly higher than the global total $(59.8 \%$, 95\% UI: $56.2-63.0 \%)$. Suboptimal breastfeeding was responsible for $39.5 \%$ of diarrhea DALYs among children under 5 (95\% UI: 27.1-52.3\%). Vitamin A and zinc deficiency were responsible for less than $10 \%$ of diarrhea DALYs among children under 5 (8 and 5\%, respectively) and were lower than the global average for these risk factors (12.9 and 6.5\%, respectively). Childhood undernutrition PAF ranged from $41.1 \%$ in the United Arab Emirates to $86.2 \%$ in Sudan. Diarrhea standardized exposure variables (SEV), a measure of the prevalence-weighted risk of diarrhea, was highest in Sudan, Afghanistan, and Yemen and lowest in the UAE, Jordan, and Kuwait.

\section{Discussion}

Our study is the most comprehensive assessment of diarrheal disease burden and the contributions of specific pathogens and risk factors in the EMR to date. In 2015, the estimated diarrhea-associated deaths and DALYs showed a slight decline from the GBD 2013 estimates of 125,000 and nearly 10 million, respectively. We also continue to find significant variation within the region, with LICs and MICs experiencing social unrest bearing the vast majority of diarrheal burden. Our data clearly illustrate the gross health inequity in the region: the HICs experienced a nominal diarrhea burden compared to the substantial burden in all LICs and some MICs.

There were no major methodological changes in diarrhea mortality modeling between GBD 2013 and GBD 2015 but there were updates to the cause of death and nonfatal data. A major difference between GBD 2013 and 2015 was the introduction of the molecular diagnostic case definition for diarrheal etiologies (Liu et al. 2014; GBD 2015 Mortality and Causes of Death Collaborators 2016; GBD 2015 Risk Factors Collaborators 2016; Kassebaum et al. 2016). In the current round of GBD, we used a systematic reanalysis of the Global Enteric Multicenter Study (GEMS) that retested roughly half of the original 22,000 moderate-to-severe stool samples with quantitative polymerase chain reaction (qPCR) (Liu et al. 2016). In transitioning to a molecular diagnostic case definition, we attribute a much larger proportion of diarrhea cases and deaths to etiologies compared to previous rounds of GBD that used non-molecular diagnostics.

The Socio-demographic Index was designed to be a measure of the socio-demographic status of a country and is well correlated with the Human Development Index (GBD 2015 Mortality and Causes of Death Collaborators 2016). Still, dramatic changes in development such as 
Fig. 1 Diarrhea mortality rate per 100,000 population, by age, 1990-2015. The mortality rate in the Eastern Mediterranean Region is shown for six age groups over time from 1990 to 2015. Points represent mean estimates and error bars represent $95 \%$ uncertainty intervals (Global Burden of Disease 2015 study, Eastern Mediterranean Region, 1990-2015)

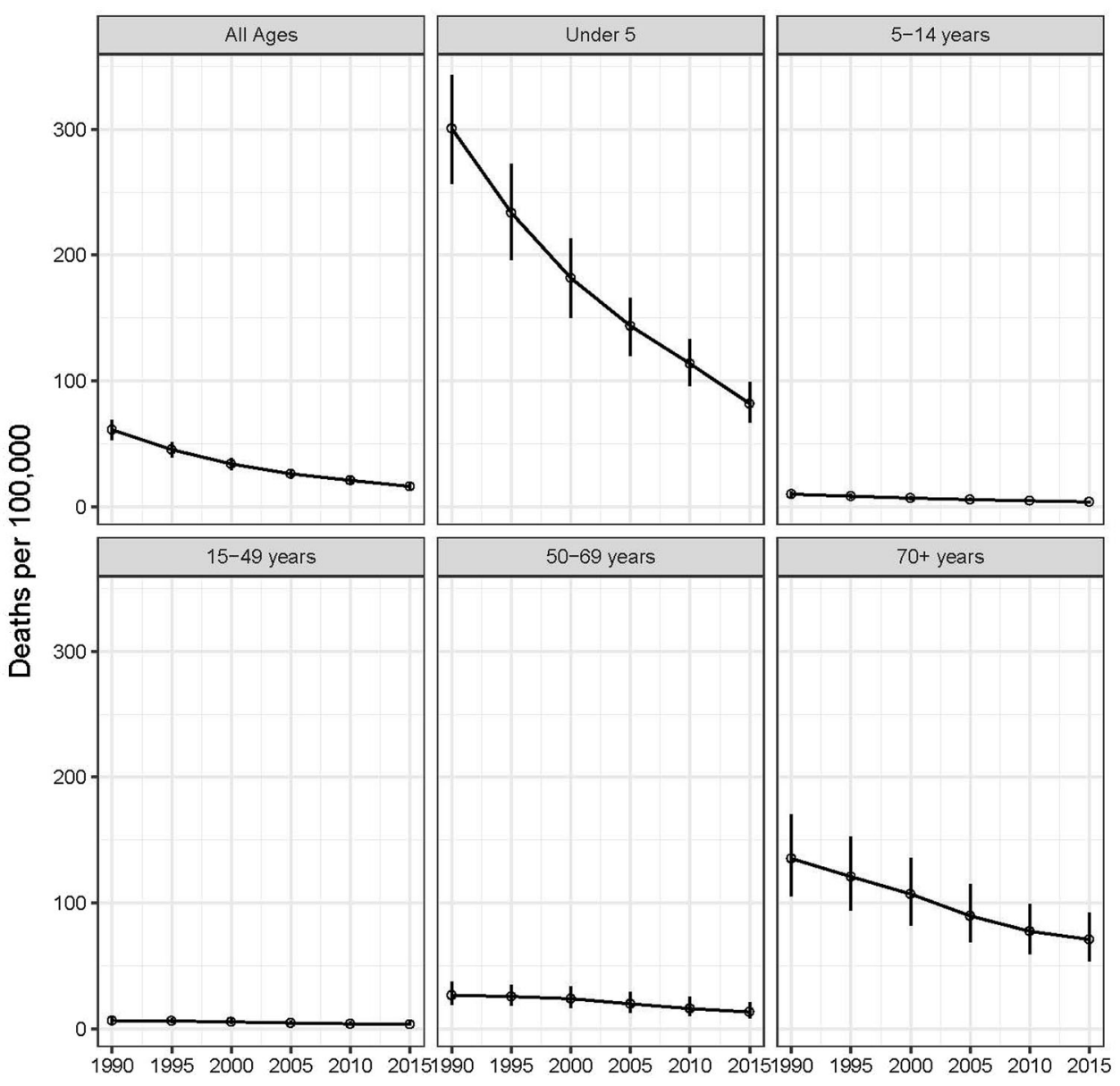

those that have occurred since the start of political unrest in some of the EMR countries, such as the Syrian civil war, may be missed or at least result in a lag in the components that make up the SDI including income, fertility, and education. We have previously shown that the SDI is a good marker of disease burden, but it may not entirely capture major sociopolitical upheavals (GBD 2015 Mortality and Causes of Death Collaborators 2016).

The introduction of vaccines against diarrheal pathogens may exacerbate inequalities in diarrhea burden. For example, although rotavirus infection was the largest contributor to the diarrheal burden of disease, in some countries in the region, rotavirus vaccine is only available in the private market. This means that wealthier families, who have less need for the vaccine, will gain the primary benefit from its availability. This is troubling because economic analyses of rotavirus vaccine introduction among a number of EMR countries have uniformly suggested that vaccine introduction would be cost-beneficial from a societal perspective (Ortega et al. 2009; Connolly et al. 2012; Javanbakht et al. 2015). One study in Somalia (the only LIC country) suggested that introduction of rotavirus as a special immunization program during a complex
Fig. 2 Diarrhea mortality among children under 5 in the Eastern Mediterranean Region, 2015. a The diarrhea mortality rate per 100,000 among children under 5 in 2015 . b Ratio of the observed under 5 diarrhea mortality rate in 2015 to the expected mortality rate based on the Socio-demographic Index only. Values below 1 indicate that the diarrhea mortality rate is lower than would be expected based on the global relationship between mortality and SDI, and values above 1 indicate higher mortality rates than would be expected (Global Burden of Disease 2015 study, Eastern Mediterranean Countries, 2015)

humanitarian emergency would meet WHO cost-effectiveness benchmarks (Gargano et al. 2015).

One unique contribution of this analysis is the inclusion of all age groups. Due to the high disease burden in young children, nearly all diarrhea interventions and most diarrhea burden studies are limited to those under 5 years of age (Boschi-Pinto et al. 2008; Fischer Walker et al. 2012; Walker et al. 2013). However, the burden among those over age 70 is substantial, with diarrheal disease-associated deaths totaling nearly one sixth of the number among those under 5. The elderly may face increased diarrhea risk due to immunosenescence and comorbidities, which may also necessitate special consideration in their treatment (Trinh 
(A)

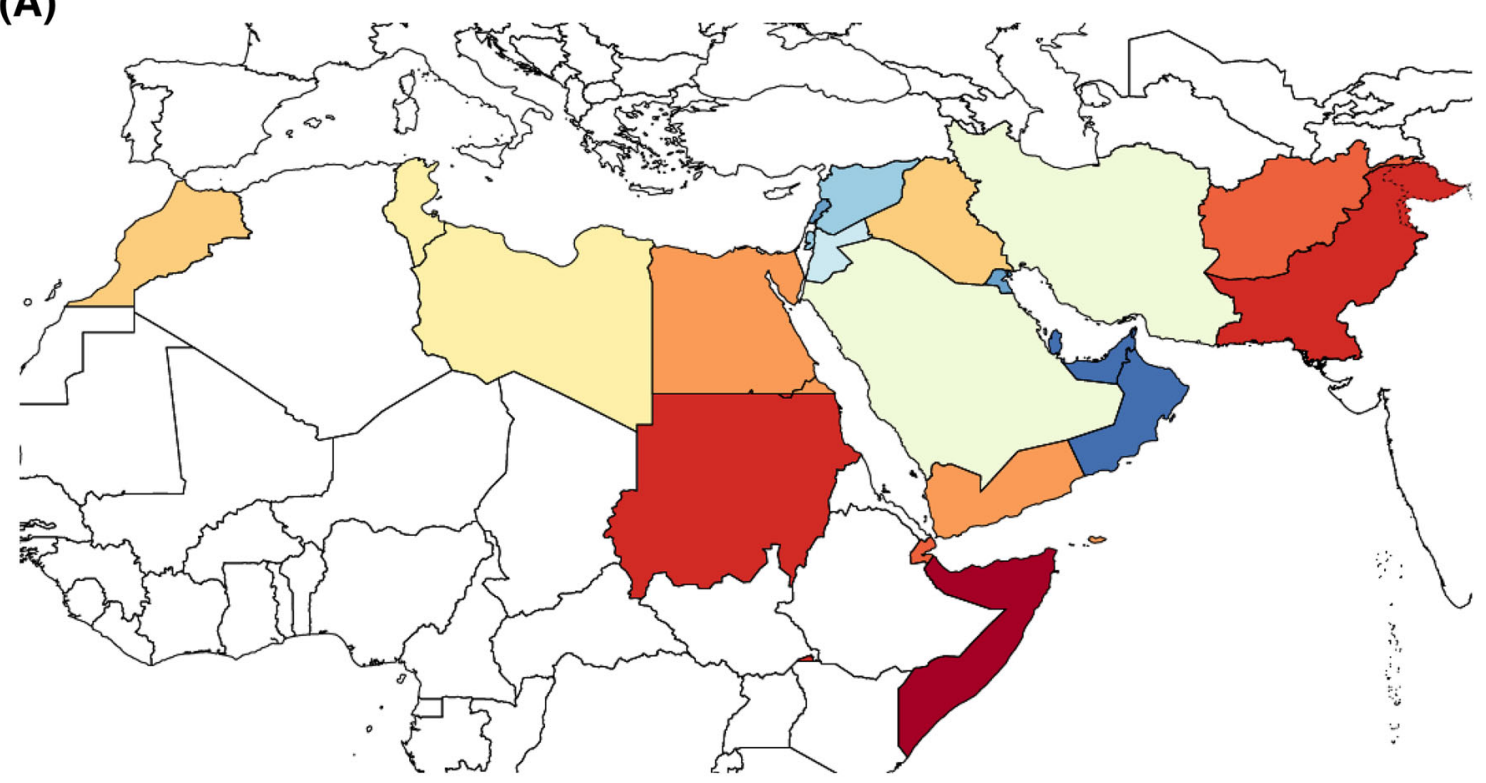
- 0 to 0.5
3.7 to 7.1
0.5 to 1
7.1 to 30.5
1 to 1.8
30.5 to 112.5
1.8 to 2.4
2.4 to 3.2
3.2 to 3.7
- 112.5 to 124.6
- 124.6 to 449.6
- 449.6 to 600

(B)

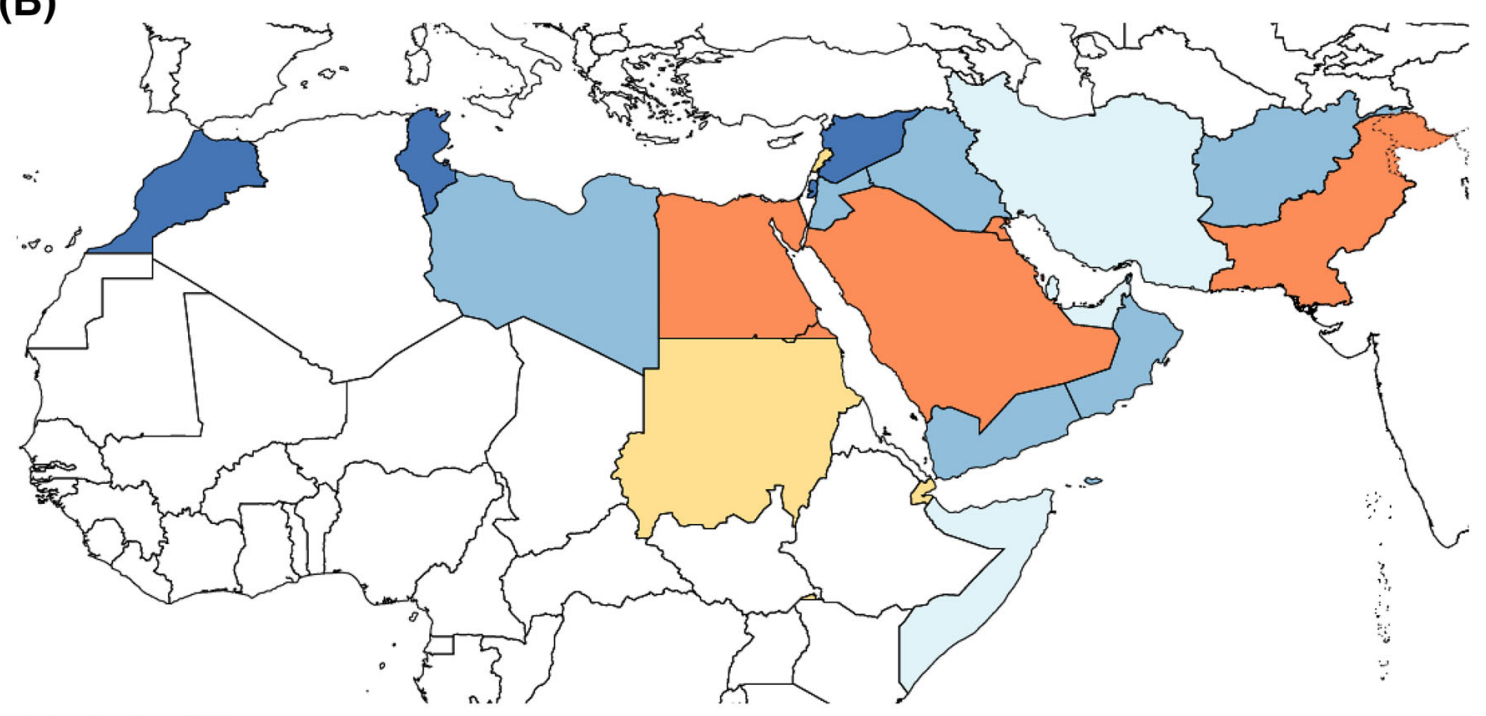
- 0 to 0.2
0.2 to 0.4
0.4 to 0.6
0.6 to 0.8
0.8 to 1
1 to 5 


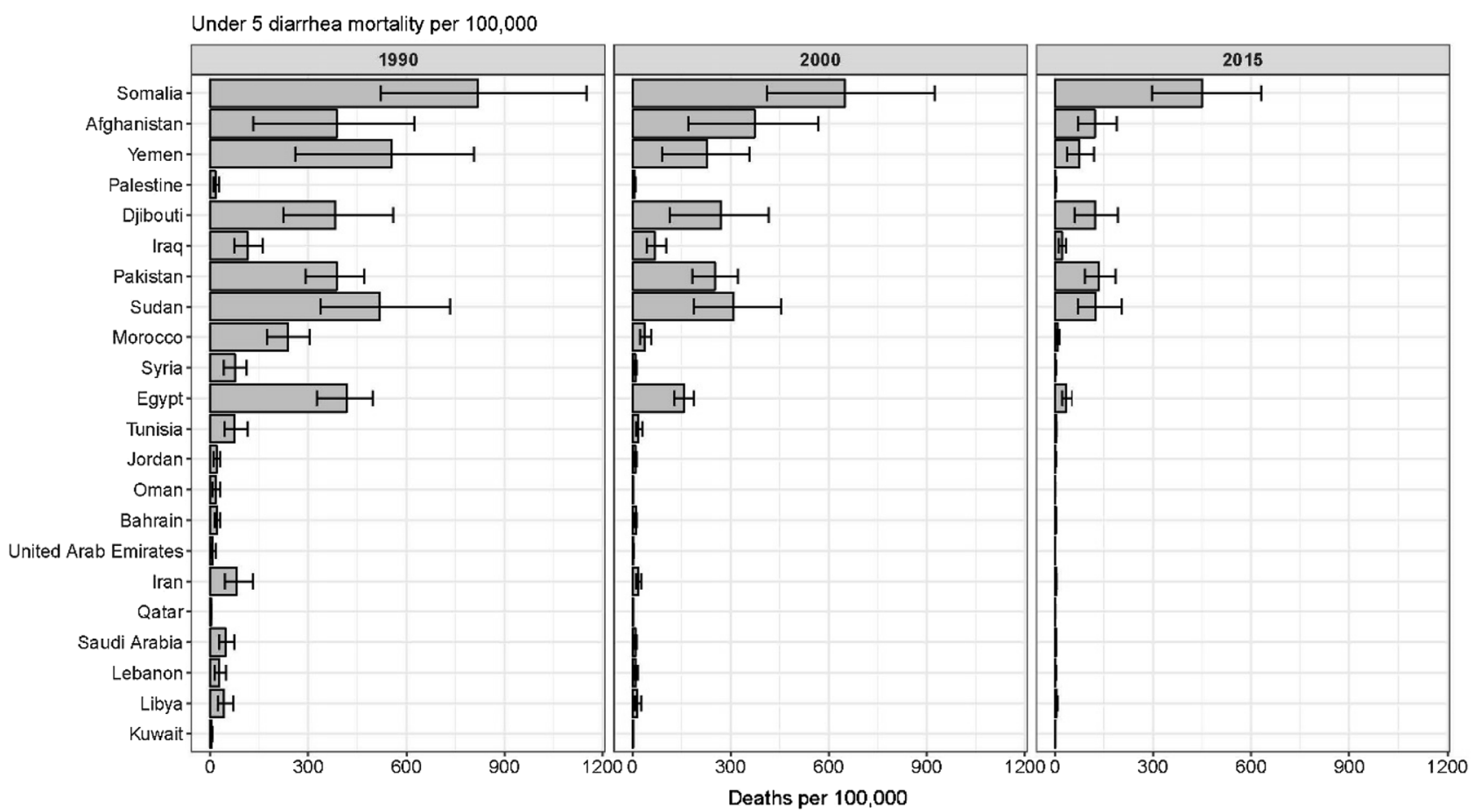

Fig. 3 The diarrhea mortality rate per 100,000 for the Eastern Mediterranean Region by country among children under 5 in 1990, 2000 , and 2015. Countries are ordered from top to bottom from lowest Socio-demographic Index (Somalia) to highest SDI (Qatar) based on
SDI values in 2015. Error bars illustrate the $95 \%$ uncertainty interval for the estimates (Global Burden of Disease 2015 study, Eastern Mediterranean Countries, 2015)

Table 3 The number of diarrhea deaths and the population attributable fraction (PAF) for 13 diarrheal etiologies among under-5 and all ages in the Eastern Mediterranean Region, 2015 (Global Burden of Disease 2015 study, Eastern Mediterranean Region, 2015)

\begin{tabular}{|c|c|c|c|c|}
\hline \multirow[t]{2}{*}{ Etiology } & \multicolumn{2}{|l|}{ Under 5} & \multicolumn{2}{|l|}{ All ages } \\
\hline & Deaths & PAF & Deaths & PAF \\
\hline Adenovirus & 4392.37 (1553.83 to 9530.86$)$ & $6.69(2.47$ to 13.85$)$ & 5285.33 (1885.13 to $11,110.37)$ & $5.1(1.87$ to 10.34$)$ \\
\hline Aeromonas & 3850.51 ( 0 to $29,059.34)$ & $\begin{array}{l}5.83(-35.98 \text { to } \\
42.15)\end{array}$ & $15,315.61(0$ to $-44,627.46)$ & $\begin{array}{l}14.72(-12.52 \text { to } \\
41.55)\end{array}$ \\
\hline Amoebiasis & 1446.31 (0 to $12,799.8)$ & $2.19(-10.65$ to 20.4$)$ & 3075.66 (0 to $17,604.66)$ & $2.97(-3.01$ to 17.06$)$ \\
\hline Campylobacter enteritis & $5478.32(1421.6$ to $11,408.45)$ & $8.3(2.35$ to 16.53$)$ & $5878.95(1504.27$ to $12,465.87)$ & $5.66(1.51$ to 11.74$)$ \\
\hline Cholera & $5142.08(2784.31$ to 9223.41$)$ & $7.86(4.27$ to 14.08$)$ & $9682.46(5614.73$ to $16,600.13)$ & $9.38(5.37$ to 16.13$)$ \\
\hline Clostridium difficile & 8.24 (4.89 to 13.04$)$ & 0.01 (0.01 to 0.02$)$ & 12.59 (8.99 to 17.62$)$ & 0.01 (0.01 to 0.02$)$ \\
\hline Cryptosporidiosis & 4569.06 (801.68 to $11,545.02)$ & $6.92(1.26$ to 16.99$)$ & $4796.2(699.01$ to $12,482.23)$ & $4.62(0.71$ to 11.52$)$ \\
\hline $\begin{array}{l}\text { Enteropathogenic } E \text {. coli } \\
\text { infection }\end{array}$ & $1639.06(132.28$ to 4608.33$)$ & $2.49(0.21$ to 6.85$)$ & $1708.03(141.29$ to 4833.27$)$ & $1.64(0.14$ to 4.68$)$ \\
\hline Enterotoxigenic $E$. coli infection & $6885.39(2931.74$ to $12,386.47)$ & 10.47 (4.76 to 18.53$)$ & $\begin{array}{l}11,102.89(5152.14 \text { to } \\
20,035.61)\end{array}$ & 10.7 (4.92 to 18.28$)$ \\
\hline Norovirus & 2402.25 (769.72 to 5439.75$)$ & $3.64(1.26$ to 8.03$)$ & 3935.54 (1027.17 to 8670.77$)$ & $3.78(1.06$ to 8.32$)$ \\
\hline Other salmonella infections & $5559.16(1663.31$ to $12,323.64)$ & $8.46(2.57$ to 18.31$)$ & 8752.77 (3002.34 to $18,462.75)$ & $8.43(3.01$ to 17.61$)$ \\
\hline Rotaviral enteritis & $13,180.3(9807.01$ to $17,738.4)$ & 20.08 (16.37 to 25.56$)$ & $\begin{array}{l}14,454.5(10,983.35 \text { to } \\
18,974.79)\end{array}$ & $13.96(11.13$ to 17.68$)$ \\
\hline Shigellosis & $\begin{array}{l}10,963.91(5480.23 \text { to } \\
19,387.69)\end{array}$ & 16.65 (9.02 to 28.57$)$ & $\begin{array}{l}19,450.5(10,025.55 \text { to } \\
33,996.23)\end{array}$ & 18.71 (10.32 to 30.61$)$ \\
\hline
\end{tabular}


and Prabhakar 2007). The increasing cholera burden in five EMR countries (Jordan, Palestine, Syria, Bahrain, and Oman) is a cause for concern, as neighboring countries remain at high risk of transmission due to the presence and movement of refugee populations among them.

We found no systematic difference in under-5 diarrhea deaths or DALYs when comparing females to males. A hypothesis that there may exists evidence of differential diarrhea mortality burden by sex is supported by a previous analysis of global demographic and health survey (DHS) data. This analysis suggested that reported that girls 1-4 years old, particularly in the Middle Eastern crescent, are at a mortality disadvantage compared to boys (Hill and Upchurch 1995). This could be explained by differences in health care access and nutritional status. Furthermore, an Egyptian study found some evidence that, even when parents sought care for their daughters with diarrhea, regional health care providers provided biased treatment in favor of boys (Yount 2003). In contrast, other studies, such as a 2009 verbal autopsy study in Iraq, found no difference in under-5 mortality by sex (Awqati et al. 2009).

\section{Public health consequences of emergencies in the region}

The EMR is facing numerous health challenges as a result of previous wars, and recent revolutions and the wars that followed (Mokdad et al. 2016; Charara et al. 2017) resulted in a huge refugee problem with millions of refugees and massive consequences for the health and well-being of millions of displaced people. The EMR now carries the largest burden of displaced populations globally.

Out of a total of 50 million refugees and internally displaced persons (IDPs) worldwide, more than 29 million (9 million refugees and 20 million IDPs) came from the region (WHO EMRO 2015). Syria is currently the world's largest source of refugees and IDPs, with more than $40 \%$ of the population now displaced both inside the country and in neighboring states. Afghanistan and Somalia face two of the longest-spanning refugee situations, with Afghanis constituting the second-largest refugee group in the world, and Somalia facing one of the world's most complex refugee situations (WHO EMRO 2015). Over the past few years, the region saw massive internal displacement in Iraq, with more than 3 million people fleeing their homes since June 2014, and in Yemen, where more than 2.3 million people have been internally displaced since March 2015 (ACAPS 2015).

The impact of these emergencies on public health is profound and enduring, affecting both the displaced populations and host communities. The risk of DD is increased in these settings due to limited access to safe water, rotavirus vaccines, and oral rehydration salts, along with other factors like infectious diseases, acute malnutrition, and inappropriate infant and young child feeding. The lack of safe drinking water as well as adequate sanitation and hygiene is especially concerning as a major risk factor for DD. Provision of a safe water supply, sanitation, and hygiene is a crucial priority, even in these emergency situations.

\section{Study limitations}

Our estimates of diarrhea mortality, morbidity, and etiology attribution are limited by data availability, and although our modeling process seeks to make use of all available data, the number of relevant publications in the region is limited and unbalanced between countries. The hierarchical modeling approach allows us to "borrow" strength across time and geography to generate the best possible estimates. Second, since we only account for the acute phase of diarrhea in our YLD estimates, the resulting DALYs severely underestimate diarrhea-associated morbidity. In future GBD updates, we expect to include longterm sequelae such as stunting and cognitive impairment, (Moore et al. 2010; MacIntyre et al. 2014; Colombara et al. 2016) which will better estimate the true burden of disease.

Despite these limitations, this analysis also has several strengths. GBD methodology ensures internal consistency so that morbidity and mortality cannot be simultaneously ascribed to competing causes and allows for comparability between countries and across regions.

\section{Conclusion}

Increased momentum of public health efforts is needed to reduce the burden of diarrhea in the EMR, especially in lower-income countries and countries experiencing political and social unrest.

Health inequities revealed in our study show that a coordinated approach that involves prevention and treatment is needed to address the multiple causes of diarrheal diseases. Regional health systems need to be strengthened to achieve the widespread availability and use of oral rehydration salts, improved nutrition, better sanitation and hygiene, and increased coverage of rotavirus immunization. Improved rates of breastfeeding should be strongly emphasized as one of the effective tools to prevent DD in countries' public health programs.

Due to the high relevance of rotavirus infections in the EMR (Malek et al. 2010), there is also an urgent need to accelerate the rollout of rotavirus vaccine in the region through government immunization programs that would ensure access for the children who are most in need. In addition, regional governments should begin deliberation 
to integrate Shigella, ETEC, and other diarrheal vaccines that are currently in preclinical and clinical trials into their Expanded Programs on Immunization (EPI) as soon as they are approved and licensed.

GBD 2015 Eastern Mediterranean Region Diarrhea Collaborators: Ali H. Mokdad, $\mathrm{PhD}$ (corresponding author), Institute for Health Metrics and Evaluation, University of Washington, Seattle, Washington, United States. Ibrahim Khalil MD, Institute for Health Metrics and Evaluation,University of Washington, Seattle, Washington, United States. Charbel El Bcheraoui, PhD, Institute for Health Metrics and Evaluation, University of Washington, Seattle, Washington, United States. Raghid Charara, MD, American University of Beirut, Beirut, Lebanon. Maziar Moradi-Lakeh, MD, Department of Community Medicine, Preventive Medicine and Public Health Research Center, Gastrointestinal and Liver Disease Research Center (GILDRC), Iran University of Medical Sciences, Tehran, Iran. Ashkan Afshin, MD, Institute for Health Metrics and Evaluation, University of Washington, Seattle, Washington, United States. Nicholas J. Kassebaum, MD, Institute for Health Metrics and Evaluation, University of Washington, Seattle, Washington, United States, Department of Anesthesiology \& Pain Medicine, Seattle Children's Hospital, Seattle, Washington, United States. Michael Collison, BS, Institute for Health Metrics and Evaluation, University of Washington, Seattle, Washington, United States. Adrienne Chew, ND, Institute for Health Metrics and Evaluation, University of Washington, Seattle, Washington, United States. Kristopher J. Krohn, BA, Institute for Health Metrics and Evaluation, University of Washington, Seattle, Washington, United States. Farah Daoud, BA/BS, Institute for Health Metrics and Evaluation, University of Washington, Seattle, Washington, United States. Danny Colombara, $\mathrm{PhD}$, Institute for Health Metrics and Evaluation, University of Washington, Seattle, Washington, United States. Kyle J. Foreman, $\mathrm{PhD}$, Institute for Health Metrics and Evaluation, University of Washington, Seattle, Washington, United States, Imperial College London, London, United Kingdom. William W. Godwin, BS, Institute for Health Metrics and Evaluation, University of Washington, Seattle, Washington, United States. Michael Kutz, BS, Institute for Health Metrics and Evaluation, University of Washington, Seattle, Washington, United States. Mojde Mirarefin, MPH, Institute for Health Metrics and Evaluation, University of Washington, Seattle, Washington, United States; Hunger Action Los Angeles, Los Angeles, CA, United States. Puja C. Rao, MPH, Institute for Health Metrics and Evaluation, University of Washington, Seattle, Washington, United States. Robert Reiner Jr, PhD, Institute for Health Metrics and Evaluation, University of Washington, Seattle, Washington, United States. Christopher Troeger, MPH, Institute for Health Metrics and Evaluation, University of Washington, Seattle, Washington, United States. Haidong Wang, $\mathrm{PhD}$, Institute for Health Metrics and Evaluation, University of Washington, Seattle, Washington, United States. Haftom Niguse Abraha, MS, Mekelle University, Mekelle, Ethiopia. Remon AbuElyazeed, PhD, GlaxoSmithKline-Vaccines, Philadelphia, PA, United States. Laith J. Abu-Raddad, PhD, Infectious Disease Epidemiology Group, Weill Cornell Medical College in Qatar, Doha, Qatar. Aliasghar Ahmad Kiadaliri, PhD, Department of Clinical Sciences Lund, Orthopedics, Clinical Epidemiology Unit, Lund University, Lund, Sweden. Alireza Ahmadi, PhD, Kermanshah University of Medical Sciences, Kermanshah, Iran. Muktar Beshir Ahmed, MPH, College of Health Sciences, Department of Epidemiology, ICT and e-Learning Coordinator, Jimma University, Jimma, Ethiopia. Khurshid Alam, $\mathrm{PhD}$, Murdoch Childrens Research Institute, The University of Melbourne, Parkville, Victoria, Australia, The University of Melbourne, Melbourne, VIC, Australia, The University of Sydney, Sydney, NSW, Australia. Reza Alizadeh-Navaei, PhD, Gastrointestinal Cancer Research Center, Mazandaran University of Medical Sciences, Sari, Iran. Rajaa Al-Raddadi, PhD, Joint Program of Family and Community
Medicine, Jeddah, Makkah, Saudi Arabia. Khalid A. Altirkawi, MD, King Saud University, Riyadh, Saudi Arabia. Nelson Alvis-Guzman, $\mathrm{PhD}$, Universidad de Cartagena, Cartagena de Indias, Colombia. Nahla Anber, PhD, Mansoura University, Mansoura, Egypt. Palwasha Anwari, MD, Self-employed, Kabul, Afghanistan. Tesfay Mehari Atey, MS, Mekelle University, Mekelle, Ethiopia. Euripide Frinel G. Arthur Avokpaho, MPH, Institut de Recherche Clinique du Bénin (IRCB), Cotonou, Benin, Laboratoire d'Etudes et de Recherche-Action en Santé (LERAS Afrique), Parakou, Benin. Umar Bacha, PhD, School of Health Sciences, University of Management and Technology, Lahore, Pakistan. Shahrzad Bazargan-Hejazi, PhD, College of Medicine, Charles R. Drew University of Medicine and Science, Los Angeles, CA, United States; David Geffen School of Medicine, University of California at Los Angeles, Los Angeles, CA, United States. Neeraj Bedi, MD, College of Public Health and Tropical Medicine, Jazan, Saudi Arabia. Isabela M. Bensenor, PhD, University of São Paulo, São Paulo, Brazil. Adugnaw Berhane, PhD, College of Health Sciences, Debre Berhan University, Debre Berhan, Ethiopia. Pascal Obong Bessong, PhD, University of Venda, Thohoyandou, South Africa. Addisu Shunu Beyene, MPH, College of Health and Medical Science, Haramaya University, Harar, Ethiopia. Zulfiqar A. Bhutta, PhD, Centre of Excellence in Women and Child Health, Aga Khan University, Karachi, Pakistan; Centre for Global Child Health, The Hospital for Sick Children, Toronto, ON, Canada. Geoffrey Colin Buckle, MD, University of California, San Francisco, San Francisco, California, United States. Zahid A. Butt, PhD, Al Shifa Trust Eye Hospital, Rawalpindi, Pakistan. Hadi Danawi, PhD, Walden University, Minneapolis, Minnesota, United States. Amare Deribew, $\mathrm{PhD}$, Nuffield Department of Medicine, University of Oxford, Oxford, United Kingdom, KEMRI-Wellcome Trust Research Programme, Kilifi, Kenya. Shirin Djalalinia, PhD, Undersecretary for Research \& Technology, Ministry of Health \& Medical Education, Tehran, Iran. Manisha Dubey, MPhil, International Institute for Population Sciences, Mumbai, India. Aman Yesuf Endries, MPH, Arba Minch University, Arba Minch, Ethiopia. Babak Eshrati, PhD, Ministry of Health and Medical Education, Tehran, Iran, Arak University of Medical Sciences, Arak, Iran. Seyed-Mohammad Fereshtehnejad, PhD, Department of Neurobiology, Care Sciences and Society (NVS), Karolinska Institutet, Stockholm, Sweden. Florian Fischer, PhD, School of Public Health, Bielefeld University, Bielefeld, Germany. Tsegaye Tewelde Gebrehiwot, MPH, Jimma University, Jimma, Ethiopia. Harish Chander Gugnani, PhD, Departments of Microbiology and Epidemiology \& Biostatistics, Saint James School of Medicine, The Quarter, Anguilla. Randah Ribhi Hamadeh, DPhil, Arabian Gulf University, Manama, Bahrain. Samer Hamidi, DrPH, Hamdan Bin Mohammed Smart University, Dubai, United Arab Emirates. Abdullatif Husseini, PhD, Institute of Community and Public Health, Birzeit University, Birzeit, Palestine. Spencer Lewis James, MD, Denver Health/University of Colorado, Denver, CO, United States. Jost B. Jonas, MD, Department of Ophthalmology, Medical Faculty Mannheim, Ruprecht-KarlsUniversity Heidelberg, Mannheim, Germany. Amir Kasaeian, $\mathrm{PhD}$, Hematology-Oncology and Stem Cell Transplantation Research Center, Tehran University of Medical Sciences, Tehran, Iran; Endocrinology and Metabolism Population Sciences Institute, Tehran, Iran. Yousef Saleh Khader, ScD, Department of Community Medicine, Public Health and Family Medicine, Jordan University of Science and Technology, Irbid, Jordan. Ejaz Ahmad Khan, MD, Health Services Academy, Islamabad, Pakistan. Gulfaraz Khan, PhD, Department of Microbiology and Immunology, College of Medicine \& Health Sciences, United Arab Emirates University, Al Ain, United Arab Emirates. Jagdish Khubchandani, PhD, Department of Nutrition and Health Science, Ball State University, Muncie, Indiana, United States. Niranjan Kissoon, MD, University of British Columbia, Vancouver, BC, Canada. Jacek A. Kopec, PhD, University of British Columbia, Vancouver, BC, Canada. Ai Koyanagi, MD, Research and Development Unit, Parc Sanitari Sant Joan de Deu (CIBERSAM), Barcelona, 
Spain. Barthelemy Kuate Defo, PhD, Department of Social and Preventive Medicine, School of Public Health, University of Montreal, Montreal, Quebec, Canada, Department of Demography and Public Health Research Institute, University of Montreal, Montreal, Canada. Heidi J. Larson, PhD, Department of Infectious Disease Epidemiology, London School of Hygiene \& Tropical Medicine, London, United Kingdom, Institute for Health Metrics and Evaluation, University of Washington, Seattle, Washington, United States. Asma Abdul Latif, $\mathrm{PhD}$, Department of Zoology, Lahore College for Women University, Lahore, Pakistan. Raimundas Lunevicius, $\mathrm{PhD}$, Aintree University Hospital National Health Service Foundation Trust, Liverpool, United Kingdom, School of Medicine, University of Liverpool, Liverpool, United Kingdom. Hassan Magdy Abd El Razek, MBBCH, Mansoura Faculty of Medicine, Mansoura, Egypt. Mohammed Magdy Abd El Razek, MBBCH, Aswan University Hospital, Aswan Faculty of Medicine, Aswan, Egypt. Reza Majdzadeh, PhD, Knowledge Utilization Research Center and Community Based Participatory Research Center, Tehran University of Medical Sciences, Tehran, Iran. Azeem Majeed, MD, Department of Primary Care \& Public Health, Imperial College London, London, United Kingdom. Reza Malekzadeh, MD, Digestive Diseases Research Institute, Tehran University of Medical Sciences, Tehran, Iran. Peter Memiah, PhD, University of West Florida, Pensacola, FL, United States. Ziad A. Memish, MD, Saudi Ministry of Health, Riyadh, Saudi Arabia, College of Medicine, Alfaisal University, Riyadh, Saudi Arabia. Walter Mendoza, MD, United Nations Population Fund, Lima, Peru. Desalegn Tadese Mengistu, MS, College of Health Sciences, Mekelle University, Mekelle, Ethiopia. Shafiu Mohammed, PhD, Health Systems and Policy Research Unit, Ahmadu Bello University, Zaria, Nigeria, Institute of Public Health, Heidelberg University, Heidelberg, Germany. Srinivas Murthy, MD, University of British Columbia, Vancouver, BC, Canada. Josephine Wanjiku Ngunjiri, PhD, University of Nairobi, Nairobi, Kenya. Felix Akpojene Ogbo, MPH, Centre for Health Research, Western Sydney University, Sydney, New South Wales, Australia. Farshad Pourmalek, $\mathrm{PhD}$, University of British Columbia, Vancouver, British Columbia, Canada. Mostafa Qorbani, PhD, Non-communicable Diseases Research Center, Alborz University of Medical Sciences, Karaj, Iran. Amir Radfar, MD, A T Still University, Kirksville, MO, United States. Anwar Rafay, MS, Contech International Health Consultants, Lahore, Pakistan, Contech School of Public Health, Lahore, Pakistan. Vafa Rahimi-Movaghar, MD, Sina Trauma and Surgery Research Center, Tehran University of Medical Sciences, Tehran, Iran. Rajesh Kumar Rai, MPH, Society for Health and Demographic Surveillance, Suri, India. Usha Ram, PhD, International Institute for Population Sciences, Mumbai, India. David Laith Rawaf, MD, WHO Collaborating Centre, Imperial College London, London, United Kingdom, North Hampshire Hospitals, Basingstroke, United Kingdom; University College London Hospitals, London, United Kingdom. Salman Rawaf, MD, Imperial College London, London, United Kingdom. Andre M.N. Renzaho, $\mathrm{PhD}$, Western Sydney University, Penrith, NSW, Australia. Satar Rezaei, PhD, School of Public Health, Kermanshah University of Medical Sciences, Kermanshah, Iran. Gholamreza Roshandel, PhD, Golestan Research Center of Gastroenterology and Hepatology, Golestan University of Medical Sciences, Gorgan, Iran; Digestive Diseases Research Institute, Tehran University of Medical Sciences, Tehran, Iran. Mahdi Safdarian, MD, Sina Trauma \& Surgery Research Center, Tehran University of Medical Sciences, Tehran, Iran. Mohammad Ali Sahraian, MD, MS Research Center, Neuroscience Institute, Tehran University of Medical Sciences, Tehran, Iran. Payman Salamati, MD, Sina Trauma and Surgery Research Center, Tehran University of Medical Sciences, Tehran, Iran. Abdallah M. Samy, PhD, Ain Shams University, Cairo, Egypt. Juan Ramon Sanabria, MD, J Edwards School of Medicine, Marshall Univeristy, Huntington, WV, United States; Case Western Reserve University, Cleveland, $\mathrm{OH}$, United States. Benn Sartorius, PhD, Public Health Medicine, School of Nursing and Public Health, University of KwaZulu-Natal, Durban,
South Africa; UKZN Gastrointestinal Cancer Research Centre, South African Medical Research Council (SAMRC), Durban, South Africa. Sadaf G. Sepanlou, PhD, Digestive Diseases Research Institute, Tehran University of Medical Sciences, Tehran, Iran. Masood Ali Shaikh, MD, Independent Consultant, Karachi, Pakistan. Mika Shigematsu, PhD, National Institute of Infectious Diseases, Tokyo, Japan; Sandia National Laboratories, Albuquerque, New Mexico, United States. Badr H. A. Sobaih, MD, King Saud University, Riyadh, Saudi Arabia. Chandrashekhar T. Sreeramareddy, MD, Department of Community Medicine, International Medical University, Kuala Lumpur, Malaysia. Bryan L. Sykes, PhD, Departments of Criminology, Law \& Society, Sociology, and Public Health, University of California, Irvine, Irvine, CA, United States. Arash Tehrani-Banihashemi, PhD, Preventive Medicine and Public Health Research Center, Iran University of Medical Sciences, Tehran, Iran. Mohamad-Hani Temsah, MD, King Saud University, Riyadh, Saudi Arabia. Abdullah Sulieman Terkawi, MD, Department of Anesthesiology, University of Virginia, Charlottesville, VA, United States; Department of Anesthesiology, King Fahad Medical City, Riyadh, Saudi Arabia; Outcomes Research Consortium, Cleveland Clinic, Cleveland, $\mathrm{OH}$, United States. Tenaw Yimer Tiruye, MPH, Debre Markos University, Debre Markos, Ethiopia. Roman Topor-Madry, PhD, Institute of Public Health, Faculty of Health Sciences, Jagiellonian University Medical College, Kraków, Poland; Faculty of Health Sciences, Wroclaw Medical University, Wroclaw, Poland. Kingsley Nnanna Ukwaja, MD, Department of Internal Medicine, Federal Teaching Hospital, Abakaliki, Nigeria. Stein Emil Vollset, DrPH, Center for Disease Burden, Norwegian Institute of Public Health, Bergen, Norway; Department of Global Public Health and Primary Care, University of Bergen, Bergen, Norway; Institute for Health Metrics and Evaluation, University of Washington, Seattle, Washington, United States. Tolassa Wakayo, MS, Jimma University, Jimma, Ethiopia. Andrea Werdecker, PhD, Competence Center Mortality-Follow-Up of the German National Cohort, Federal Institute for Population Research, Wiesbaden, Germany. Charles Shey Wiysonge, PhD, South African Medical Research Council, Cochrane South Africa, Cape Town, South Africa; Stellenbosch University, Cape Town, South Africa. Abdulhalik Workicho, MPH, Jimma University, Jimma, Ethiopia, Ghent University, Ghent, Belgium. Mohsen Yaghoubi, MSc, School of Public Health, University of Saskatchewan, Saskatoon, Saskatchewan, Canada. Mehdi Yaseri, $\mathrm{PhD}$, Tehran University of Medical Sciences, Terhan, Iran, Ophthalmic Research Center, Shahid Beheshti University of Medical Sciences, Tehran, Iran. Muluken Yenesew, MPH, Bahir Dar University, Bahir Dar, Ethiopia. Naohiro Yonemoto, MPH, Department of Biostatistics, School of Public Health, Kyoto University, Kyoto, Japan. Mustafa Z. Younis, DrPH, Jackson State University, Jackson, MS, United States. Maysaa El Sayed Zaki, PhD, Faculty of Medicine, Mansoura University, Mansoura, Egypt. Sanjay Zodpey, PhD, Public Health Foundation of India, Gurgaon, India. Bassel Zein, MS, Neuroscience Department, Georgetown University, Washington, DC, United States. Aisha O. Jumaan, $\mathrm{PhD}$, Independent Consultant, Seattle, Washington, United States. Theo Vos, PhD, Institute for Health Metrics and Evaluation, University of Washington, Seattle, Washington, United States. Simon I. Hay, DSc, Oxford Big Data Institute, Li Ka Shing Centre for Health Information and Discovery, University of Oxford, Oxford, United Kingdom, Institute for Health Metrics and Evaluation, University of Washington, Seattle, Washington, United States. Mohsen Naghavi, $\mathrm{PhD}$, Institute for Health Metrics and Evaluation, University of Washington, Seattle, Washington, United States. Christopher J. L. Murray, DPhil, Institute for Health Metrics and Evaluation, University of Washington, Seattle, Washington, United States.

\section{Compliance with ethical standards}

Ethical approval This manuscript reflects original work that has not previously been published in whole or in part and is not under 
consideration elsewhere. All authors have read the manuscript and have agreed that the work is ready for submission and accept responsibility for its contents. The authors of this paper have complied with all ethical standards and do not have any conflicts of interest to disclose at the time of submission. The funding source played no role in the design of the study, the analysis and interpretation of data, and the writing of the paper. The study did not involve human participants and/or animals; therefore, no informed consent was needed.

Funding This research was funded by the Bill \& Melinda Gates Foundation.

Conflict of interest The authors declare that they have no conflicts of interest at this time.

Open Access This article is distributed under the terms of the Creative Commons Attribution 4.0 International License (http://crea tivecommons.org/licenses/by/4.0/), which permits unrestricted use, distribution, and reproduction in any medium, provided you give appropriate credit to the original author(s) and the source, provide a link to the Creative Commons license, and indicate if changes were made.

\section{References}

ACAPS (2015) Global emergency overview: November 2015. http:// reliefweb.int/sites/reliefweb.int/files/resources/Global\%20Emer gency $\% 20$ Overview\%20Snapshot $\% 2011 \% 20-\% 2017 \% 20$ Novem ber\%202015.pdf. Accessed 12 Jul 2017

Awqati NA, Ali MM, Al-Ward NJ et al (2009) Causes and differentials of childhood mortality in Iraq. BMC Pediatr 9:40. doi:10.1186/1471-2431-9-40

Enzley S, Barros F, UNICEF (1997) A global review of diarrhoeal disease control. https://www.unicef.org/evaldatabase/files/Glo bal_1997_A_Global_Review.pdf. Accessed 12 Jul 2017

Boschi-Pinto C, Velebit L, Shibuya K (2008) Estimating child mortality due to diarrhoea in developing countries. Bull World Health Organ 86:710-717. doi:10.1590/S004296862008000900015

Charara R, Forouzanfar M, Naghavi M et al (2017) The burden of mental disorders in the Eastern Mediterranean Region, 1990-2013. PLoS One 12:e0169575. doi:10.1371/journal.pone. 0169575

Cobb LK, Baer F, Debay M et al (1996) Final assessment of the Egypt child survival project. http://pdf.usaid.gov/pdf_docs/Pdabn167. pdf. Accessed 12 Jul 2017

Colombara DV, Khalil IA-M, Rao PC et al (2016) Chronic health consequences of acute enteric infections in the developing world. Am J Gastroenterol Suppl 3:4-11. doi:10.1038/ajgsup.2016.9

Connolly MP, Topachevskyi O, Standaert B et al (2012) The impact of rotavirus vaccination on discounted net tax revenue in Egypt: a government perspective analysis. PharmacoEconomics 30:681-695. doi:10.2165/11597750-000000000-00000

El-Rafie M, Hassouna WA, Hirschhorn N et al (1990) Effect of diarrhoeal disease control on infant and childhood mortality in Egypt. Report from the National Control of Diarrheal Diseases Project. Lancet Lond Engl 335:334-338

Fischer Walker CL, Perin J, Aryee MJ et al (2012) Diarrhea incidence in low- and middle-income countries in 1990 and 2010: a systematic review. BMC Public Health 12:220. doi:10.1186/ 1471-2458-12-220

Flaxman A, Vos T, Murray C (2015) Integrated meta-regression framework for descriptive epidemiology. University of Washington Press, Washington
Foreman KJ, Lozano R, Lopez AD, Murray CJ (2012) Modeling causes of death: an integrated approach using CODEm. Popul Health Metr 10:1. doi:10.1186/1478-7954-10-1

Forsberg BC, Petzold MG, Tomson G, Allebeck P (2007) Diarrhoea case management in low- and middle-income countries-an unfinished agenda. Bull World Health Organ 85:42-48

Gargano LM, Tate JE, Parashar UD et al (2015) Comparison of impact and cost-effectiveness of rotavirus supplementary and routine immunization in a complex humanitarian emergency. Somali case study. Confl Health 9:5. doi:10.1186/s13031-0150032-y

GBD 2015 Mortality and Causes of Death Collaborators (2016) Global, regional, and national life expectancy, all-cause mortality, and cause-specific mortality for 249 causes of death, 1980-2015: a systematic analysis for the Global Burden of Disease Study 2015. Lancet Lond Engl 388:1459-1544. doi:10. 1016/S0140-6736(16)31012-1

GBD 2015 Risk Factors Collaborators (2016) Global, regional, and national comparative risk assessment of 79 behavioural, environmental and occupational, and metabolic risks or clusters of risks, 1990-2015: a systematic analysis for the Global Burden of Disease Study 2015. Lancet Lond Engl 388:1659-1724. doi:10. 1016/S0140-6736(16)31679-8

Guerrant RL, DeBoer MD, Moore SR et al (2013) The impoverished gut - a triple burden of diarrhoea, stunting and chronic disease. Nat Rev Gastroenterol Hepatol 10:220-229. doi:10.1038/nrgas tro.2012.239

Heidt P, Lange D, Riddle M et al (2014) Persisting consequences of intestinal infection. Old Herborn Univ Semin Monogr 27:125-137

Hill K, Upchurch DM (1995) Gender differences in child health: evidence from the demographic and health surveys. Popul Dev Rev 21:127-151. doi:10.2307/2137416

Institute for Health Metrics and Evaluation Global Health Data Exchange I GHDx. http://ghdx.healthdata.org/. Accessed 24 Apr 2017

Javanbakht M, Moradi-Lakeh M, Yaghoubi M et al (2015) Costeffectiveness analysis of the introduction of rotavirus vaccine in Iran. Vaccine 33(Suppl 1):A192-A200. doi:10.1016/j.vaccine. 2014.12.035

Kassebaum NJ, Arora M, Barber RM et al (2016) Global, regional, and national disability-adjusted life-years (DALYs) for 315 diseases and injuries and healthy life expectancy (HALE), 1990-2015: a systematic analysis for the Global Burden of Disease Study 2015. Lancet 388:1603-1658. doi:10.1016/ S0140-6736(16)31460-X

Khalil I, Colombara DV, Forouzanfar MH et al (2016) Burden of diarrhea in the Eastern Mediterranean Region, 1990-2013: findings from the Global Burden of Disease Study 2013. Am J Trop Med Hyg 95:1319-1329. doi:10.4269/ajtmh.16-0339

Kotloff KL, Nataro JP, Blackwelder WC et al (2013) Burden and aetiology of diarrhoeal disease in infants and young children in developing countries (the Global Enteric Multicenter Study, GEMS): a prospective, case-control study. Lancet 382:209-222. doi:10.1016/S0140-6736(13)60844-2

Lindsay B, Saha D, Sanogo D et al (2015) Association between shigella infection and diarrhea varies based on location and age of children. Am J Trop Med Hyg 93:918-924. doi:10.4269/ ajtmh.14-0319

Liu J, Kabir F, Manneh J et al (2014) Development and assessment of molecular diagnostic tests for 15 enteropathogens causing childhood diarrhoea: a multicentre study. Lancet Infect Dis 14:716-724. doi:10.1016/S1473-3099(14)70808-4

Liu J, Platts-Mills JA, Juma J et al (2016) Use of quantitative molecular diagnostic methods to identify causes of diarrhoea in children: a reanalysis of the GEMS case-control study. Lancet 388:1291-1301. doi:10.1016/S0140-6736(16)31529-X 
MacIntyre J, McTaggart J, Guerrant RL, Goldfarb DM (2014) Early childhood diarrhoeal diseases and cognition: are we missing the rest of the iceberg? Paediatr Int Child Health 34:295-307. doi: $10.1179 / 2046905514$ Y.0000000141

Malek MA, Teleb N, Abu-Elyazeed R et al (2010) The epidemiology of rotavirus diarrhea in countries in the Eastern Mediterranean Region. J Infect Dis 202(Suppl):S12-S22. doi:10.1086/653579

Mokdad AH, Forouzanfar MH, Daoud F et al (2016) Health in times of uncertainty in the eastern Mediterranean region, 1990-2013: a systematic analysis for the Global Burden of Disease Study 2013. Lancet Glob Health 4:e704-e713. doi:10.1016/S2214109X(16)30168-1

Moore SR, Lima NL, Soares AM et al (2010) Prolonged episodes of acute diarrhea reduce growth and increase risk of persistent diarrhea in children. Gastroenterology 139:1156-1164. doi:10. 1053/j.gastro.2010.05.076

National Control Of Diarrheal Diseases Project (1988) Impact of the National Control of Diarrhoeal Diseases project on infant and child mortality in Dakahlia, Egypt. Lancet 332:145-148. doi:10. 1016/S0140-6736(88)90693-9

Ortega O, El-Sayed N, Sanders JW et al (2009) Cost-benefit analysis of a rotavirus immunization program in the Arab Republic of Egypt. J Infect Dis 200:S92-S98. doi:10.1086/605057

PATH (2017) rotavirus vaccine access and delivery. http://sites.path. org/rotavirusvaccine/country-introduction-maps-and-spread sheet/. Accessed 13 Jul 2017
The World Bank (2016) GNI per capita, PPP (current international \$). http://data.worldbank.org/indicator/NY.GNP.PCAP.PP.CD. Accessed 12 Jul 2017

Trinh C, Prabhakar K (2007) Diarrheal diseases in the elderly. Clin Geriatr Med 23:833-856, vii. doi: 10.1016/j.cger.2007.06.005

Walker CLF, Rudan I, Liu L et al (2013) Global burden of childhood pneumonia and diarrhoea. Lancet 381:1405-1416. doi:10.1016/ S0140-6736(13)60222-6

WHO (1992) Programme for control of diarrhoeal diseases: eight programme report, 1990-1991. http://apps.who.int/iris/handle/ 10665/61647. Accessed 12 Jul 2017

WHO EMRO (2015) Refugees and internally displaced persons in the Eastern Mediterranean Region: a health perspective. http://www. emro.who.int/images/stories/eha/documents/migrants_refugees_ position_paper.pdf?ua=1. Accessed 12 Jul 2017

WHO (2016) Global health observatory data repository: cholera. http://apps.who.int/gho/data/node.main.174?lang=en. Accessed 12 Jul 2017

Wickham H (2009) ggplot2: elegant graphics for data analysis. J Stat Anal 35(1). doi:10.1007/978-0-387-98141-3

Yount KM (2003) Provider bias in the treatment of diarrhea among boys and girls attending public facilities in Minia, Egypt. Soc Sci Med 1982 56:753-768 